\title{
Dynamic Resource Allocation and Priority Based Scheduling for Heterogeneous Services in Cognitive Radio Networks
}

\author{
Santhamurthy Tamilarasan ${ }^{1 *}$ \\ Kumar Parasuraman ${ }^{2}$ \\ ${ }^{I}$ Manonmaniam Sundaranar University, Tirunelveli, Tamil Nadu, India \\ ${ }^{2}$ Manonmaniam Sundaranar University, Tirunelveli, Tamil Nadu, India \\ * Corresponding author's Email: stamilarasan0606@gmail.com
}

\begin{abstract}
As a futuristic solution to the serious scarcity of spectrum and radio resources in today's scenario, cognitive radio offers great solutions. Cognitive radio is a groundbreaking approach in improving the utilization of precious natural resources. Cognitive radio network (CRN) is an intelligent wireless communication system that is aware of its environment. The problem of spectrum sensing by heterogeneous nodes, with varying computing power and sensing range, and spectrum allocation in CRN where there exist multiple primary users and secondary users have been considered. Focus has been laid on optimum resource allocations and scheduling for primary user (PU) and secondary user (SU) to maximize the performance of the whole network by reducing the interference at PU and also maximizing the throughput and minimizing the risk of overlapping the coverage of CRNs. The effectiveness of DRAPBS-CRN algorithm is compared with DRA-CRN and is found to be $26 \%$ more efficient using ns-2 simulation.
\end{abstract}

Keywords: Cognitive radio; MIMO; Femtocell; Nash equilibrium; SINR.

\section{Introduction}

\subsection{Cognitive Radio and Intelligent Antenna}

In recent years, there has been a hike in a number of internet users (accessing in a variety of ways) [1]. The licensed users (primary users), use the licensed spectrum for special purposes [2]. In practical application, the licensed spectrum has very low utilization compared to the non-licensed band. Cognitive Radio (CR) is an intelligent radio [3], that can be programmed in any given network, by using the best wireless channel available in its vicinity [4, $5,6,7]$ as one key technology to activate the utilization of spectrum resource $[8,9]$. Hence, such a Dynamic Spectrum Access improves the performance of the communication network and makes the network more reliable and robust even though the nodes are mobile and has fewer power sources $[10,11,12,13]$.

In a cognitive radio with intelligent antenna, a transmitter/receiver is designed to intelligently detect whether a particular segment of the radio spectrum is currently in use or not and to access (and out of, as necessary) the temporarily unused spectrum very rapidly, without interfering with the transmissions of other authorized users [14, 15]. Smart Antenna (also known as adaptive array antenna, multiple antennas and MIMO) are antenna arrays with smart processing algorithm used to identify special signal signature such as the direction of arrival of the signal to track and locate the antenna beam on the target $[16,17,18]$. Hence determines the best signal to be obtained with high SNR and optimum power utilization.

\subsection{Metropolitan Network and Femtocell}

A metropolitan area network (MAN) [19] is a network that interconnects users with computer resources in a geographic area or region larger than that covered by even a large local area network (LAN) but smaller than the area covered by a wide area network (WAN) [20, 21, 22]. The term is applied to the interconnection of networks in a city into a single larger network (which may then also offer efficient connection to a wide area network). [23] To increase capacity and exploit physical entities, reduction of cell size is entertained. But in recent years, it reached its limit of sustainability by the introduction of femtocell $[24,25]$. 
Femtocell [3] is a wireless access point that improves cellular reception inside a home or office building. In telecommunication, a Femtocell acts a small low-power cellular base station. In telecommunication industry use of small cell, lowpower access points operating in licensed and unlicensed spectrum, with femtocells as the subset has been used widely. A femtocell allows the service provider to extend service coverage at cell edges or indoor, especially where access would otherwise be limited or unavailable. The range of a femtocell is in the order of 10 meters.

Femtocells were originally called access point base station. They are compatible with CDMA2000, WiMAX and UMTS mobile telephony devices, using the provider's own licensed spectrum to operate. Consumer-oriented femtocells will support no more than 4 active users while enterprise-grade femtocells can support up to 16 active users. The use of femtocell in metropolitan area network (MAN) [7] can increase the efficiency of spectrum usage and power allocation.

The rest of the paper is organized as follows: In section 2, literature review, the background and related works are described, in Section 3 the problem identification along with the solution to overcome the drawbacksis described(DRAPBSCRN) and Section 4, describes the proposed DRAPBS-CRN approach. Section 5 shows the simulation result and compares DRAPBS-CRN with the existing DRA-CRN approach, and Section 6 concludes the article.

\section{Literature Review}

P. Cheng et al [1] have designed a game approach for the problem of resource allocation in an OFDMA based cell as a two-tier game. In RAGame, each PU dynamically accesses the subchannels according to his payoff while in PS-Game; each SU buys a radio from some PU to maximize his utility. Furthermore, they propose two algorithms to allocate resource among PUs and SUs in a distributed manner. Their algorithms can converge to Nash equilibrium automatically, which maximizes the utilization of the whole network resource. Besides, their relay scheme greatly improves the throughput of SUs.

Y. Tachwali et al [2] have proposed a novel resource allocation framework based on the bandwidth-power product minimization, which is an effective metric in evaluating the spectral resource consumption in a cognitive radio environment. The framework takes into consideration the challenges aforementioned. The proposal shows a significant enhancement in spectral efficiency.

$\mathrm{R}$. Xie et al [3] have proposed a spectrum sharing and resource allocation for energy-efficient heterogeneous cognitive radio networks with femtocells. They use the price of interference to model the interference between femtocells and macrocells. They formulate the problem of interference management and power allocation as a Stackelberg game. In addition, an iteration algorithm based on price updating is proposed to obtain the Stackelberg equilibrium solution to the resource allocation problem for energy efficiency.

Y. Teng et al [4] have proposed energy-efficient resource allocation in the multi-secondary user (SU) cognitive radio networks with network coding based cooperative transmission (NcCT). They set up a framework for multi-SU resource allocation game with Nash bargaining solution (NBS) under the cognitive radio scenario (CR-MSU-NBS game). These are the sum of pairwise NBS function with pairing strategy is exploited as the network optimization objective and context conditions as constraints.

J. M. Alfonso and L. B. Agudelo [7] present a new approach of Spectrum Sensing using a Compressive Sensing technique named Finite Rate of Innovation in a Cognitive Radio Network with centralized Spectrum Management based Spectrum Broker in the next generation wireless communications networks. The use of compressive sensing techniques improves the performance of the control channel in cognitive radio due the traffic control protocol requires smaller packet sizes. The performance of the cognitive network in function of the control packet size was determinate by analysis of collisions when different secondary users trying to access spectrum resources and they make the request to the Spectrum Broker. They observed that there are fewer collisions between control packets and collision probability is smaller if the compressive technique is used, thus improving the performance in a fair resource allocation for cognitive radio networks.

C. Gao et al [8] have mathematically modeled the opportunities and constraints for CR network with the objective of maximizing the weighted network throughput. They proposed a centralized algorithm and a distributed algorithm to flexibly assign spectrum channel or spatial DoF exploiting the multiuser diversity, channel diversity and spatial diversity for a higher performance in a practical network. The algorithm further supports different transmission priorities, reduces transmission delay and ensures fair transmissions among nodes by 
providing all nodes with certain transmission probability. Results show that their algorithm is very effective and can significantly increase the network throughput while reducing the delay.

D. G"oz" upek and F. Alag"oz [9] have proposed a very general scheduling model achieving goals such as making frequency, time slot, and data rate allocation to secondary users with possibly multiple antennas, in a heterogeneous multi-channel and multi-user scenario. Their schedulers ensure that reliable communication between the cognitive base station and secondary users. They also propose a heuristic algorithm for the fair schedulers. Their performance is in terms of both total throughput and fairness for varying number of secondary users, frequencies, antennas, and window size.

\section{Problem Identification and Solution}

From the literature review, it can be concluded that there is no work which jointly provides resource allocation and scheduling in CRN. Considering networks with heterogeneous services would be difficult in allocating the resources and assign the channel to each node in the given network. In [12], only the secondary users with different rate requirements are considered ignoring the delay requirements. Moreover, while estimating the channel condition for efficient communication, the channel quality indicator (CQI) has to be considered.

In this proposal, we propose to develop a dynamic resource allocation and priority-based scheduling for heterogeneous services in cognitive radio network (CRN). The network consists of primary users (PU) and secondary users (SU) with different service requirements in a heterogeneous network. The secondary base station (BS) is responsible for resource allocation for different SU's [12] present in the network. The SU's can be categorized as: SU with minimum-rate guarantee (MRG) [12], SU with minimum delay guarantee (MDG), SU with minimum rate and delay guarantee (MRDG) and SU with best effort service (BE).

While allocating resources to the set of SU, the objective function should satisfy the following constraints:

(i) Total power constraint [12] in which the total transmit power of SU on all channels should be within the power budget at the secondary BS.

(ii) For MRG, the transmission rate for $\mathrm{SU}$ should be greater than the minimum-rate threshold [12]

(iii) For MDG, the transmission delay for SU should be less than the deadline threshold. (iv) For MRDG, the transmission rate should be greater than the minimum-rate threshold and the transmission delay should be less than the deadline threshold.

(v) For BE, fairness constraint should be satisfied.

Here a channel quality indicator (CQI) is used as a utility function [13] for each channel. The CQI is estimated in terms of the signal-to-interference-plusnoise ratio (SINR). For each incoming stream, the priority of packets is determined depending on the service type and queuing delay [8]. Then an objective function is estimated for each stream by multiplying the priority with channel gain. Then the streams can be sorted in the descending order of this objective values, then allocated to the respective type of SU. The stream with highest objective function is allocated to the MRDG, followed by MRG and MDG. The stream with least objective function is assigned to $\mathrm{BE}$.

\section{Proposed Solution}

\subsection{System Model}

In cognitive radio communication systems, users are dived into primary users and secondary users (secondary users). The secondary users (cognitive users) have a relative low spectrum access authority. Secondary users must continuously sense the environment and must be aware of any changes in their wireless network and make adjustments to communication changes accordingly without causing harmfulinterference to the primary users. Here as already know the primary users represent the licensed spectrum users whereas the intelligent cognitive users (secondary users) represent the users accessing the unlicensed spectrum for communication. The main objective of the spectrum sensing is to find available free spectrum resources (un-accessed channels), estimate the channel quality, transmit the data packets and design self-adaptive transmitting waveform which fit spectrum characteristics based on communication needs. When the channels are not accessed by the primary users the secondary users can access the idle channel optimally. And periodically check the ability of the PU such that the Cognitive users' handovers the channel to the PU as soon as a PU is detected. Hence, the SU doesnot interferes with the PU and only access the idle channel when the PU is not accessing the given channel. PUs operations are given more priority as during the channel utilization there must not be any interference experienced by 
the PU (licensed user like-Satellite communication, Medical Equipments).

Assume that there are common control units (SU-BS) (Secondary Base station) to coordinate the resource allocation for SUs. As soon as SUs requests the SU-BS for a channel. The SU-BS would determine "which channel to access" and "which SU to be provided the channel?".

Here in this paper the heterogeneous SUs are considered:

- SU with minimum-rate guarantee (MRG) [12],

- SU with minimum delay guarantee (MDG),

- SU with minimum rate and delay guarantee (MRDG)

- $\quad$ SU with best effort service (BE).

We simply assume $K_{l}$ SUs with minimum-rate guarantee (MRG), $K_{2} \mathrm{SUs}$ with minimum delay guarantee (MDG), $K_{2} \mathrm{SUs}$ with minimum rate and delay guarantee (MRDG) and $K_{2} \mathrm{SU}$ with best effort service (BE). We also assume $N$ ideal sub-channels in a given time slot. The number $K_{1}, K_{2}, K_{3}, K_{4}$ and $\mathrm{N}$ can vary dynamically in different time slots.

In this paper to reduce the complexity, we have considered $k_{1}, k_{2}, k_{3}$, and $k_{4}$ and relation defined for our approach as, $K_{I}=K_{2}=K_{3}=K_{4}$. Hence we consider that there are equal number of heterogeneous SUs with MRG, MDG, MDRG and BE.

The following assumptions are made for optimal resource allocation in the given network with heterogeneous SUs and multiple PUs:

- Each sub-channels occupancy must be represented as a binary index $\gamma_{k, n} \in\{0,1\} \cdot \gamma_{k, n}$ denotes the sub channel $\mathrm{n}$ accessed by SU $\mathrm{k}$. If the sub-channel is free $\gamma_{k, n}=0$ whereas if the channel is busy $\gamma_{k, n}=1$.

- Total power constraint: Let $P_{\text {TOTAL }}$ denote the total power available for the SUs. $P_{k, n}$ denote the transmit power for $\mathrm{SU} \mathrm{k}$ in sub-channel $\mathrm{n}$.

$$
\sum_{k=1}^{k_{1}+k_{2}+k_{3}+k_{4}} \sum_{n=1}^{N} P_{k, n} \gamma_{k, n} \leq P_{\text {TOTAL }}
$$

(For example: -consider a license-free frequency band

865-867 MHz

Use: low power RFID equipment or any other low power wireless device or equipment power

Max. Output power: 1 Watt

Carrier Bandwidth: $200 \mathrm{KHz}$ )

- Minimum rate guarantee: Let $R_{K_{\min }}$ denote the minimum rate requirement for SU $k$.

$$
R_{k} \geq R_{K_{\text {min }}} \quad \forall k \in k_{1} \cup k_{2} \cup k_{3} \cup k_{4}
$$

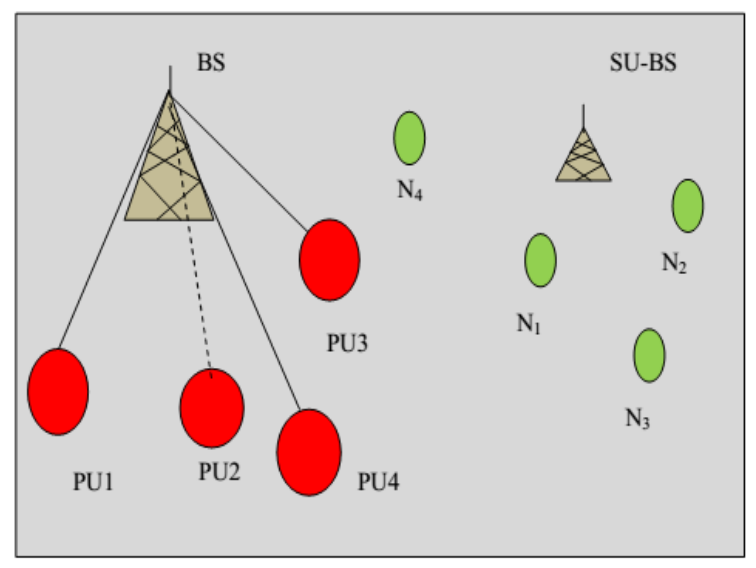

Figure.1 Heterogeneous cognitive radio network with multiple PU and SU with idle $\mathrm{PU}_{2}$ Channel $\left(\mathrm{p}_{\mathrm{r}}=0\right)$

At the SU-BS, the presence of the primary user is estimated periodically using the utility function Channel Quality Indicator (CQI). CQI also quantifies the utility that each channel offers to the $\mathrm{CR}$ in terms of average information transfer capacity and the interference. As soon as the CQI value decreases beyond a threshold value, it denotes the presence of PU and hence the SU handsover the sub-channel back to the PU.

Let us consider $\psi$ number of sub-channels in this cell. Then the set of sub-carries can be represented as, $C=\left\{C_{1}, \ldots C \varphi\right\}$. The Total bandwidth of the cell is $B$ and each sub contains the same quality of sub-carriers. Hence, the bandwidth of each sub-channel can be given by $\frac{\beta}{\varphi}$.

$C Q I$ of any sub-channel is defined using the Signal-to-Interference-Plus-Noise Ratio (SINR) detected by the SU-BS during channel allocation and during channel accessing.

Signal-to-Interference-Plus-Noise Ratio (SINR) is a quantity used to represent the channel capacity (or rate of information being transferred) in a wireless communication system such as networks. SINR is defined as the power of interested signal divided by the sum of interference power (from all the interfering signals) and the power of arbitrary background noise.

$$
\text { SINR }=\frac{\text { PSignal }}{\text { PInterference }+ \text { NOISE }}
$$

$P_{\text {Interference }}$ represents the interference power of other signals in the network. $P_{\text {signal }}$ represents the power of the incoming signal of interest. If the power of background noise term $P_{\text {Interference }}=0$ then the SINR is reduced to Signal to Signal-to-Noise Ratio (SNR). 
Channel Quality Indicator can be measured at SUBS by,

$$
C Q I=\log _{2}(1+S I N R)
$$

By threshold value at the SU-BS, the value of $C Q I$ estimated can be monitored and as soon the SU request for a channel the $C Q I$ value of the channel is estimated and the assigned to the SU only if the $C Q I>$ threshold, else the channel is not assigned to the SU. After the channel is assigned to SU in between the data transmission SU-BS finds degradation in $C Q I$ showing the presence of $\mathrm{PU}$, the SU hands-off the channel back to the PU as shown in fig. 2.

Based on Dynamic Spectrum Allocation for cognitive radio, when a primary user (PU) returns to the band area which cognitive users are using,cognitive radio have to pause the work and switch to another band, this is called spectrum handoff. The switching needs to be done as smooth as possible because the sudden transition of working spectrum may lead to decreased transmission reliability or even transmission failure.

As shown in fig. 2, The spectrum handoff takes place from PU to the secondary user and vice versa. For example in the time slot $t_{1}$ frequency range $f_{1}$ to $\mathrm{f}_{2}$ is vaccant and hence the $\mathrm{SU}$ which requests the spectrum slot is allocated the same by the SU-BS and the reset of the frequency slots (e.g- $f_{3}$ to $f_{4}$ ) are being accesed by some other PUs and hence those chennels are unintrupted. Whereas in the time slot $t_{2}$ to $t_{3}$ both the frequency slots $f_{2}$ to $f_{3}$ and $f_{3}$ to $\mathrm{f}_{4}$ is vacant and hence can be accesed by the SU. (Illustration for section 3.1)

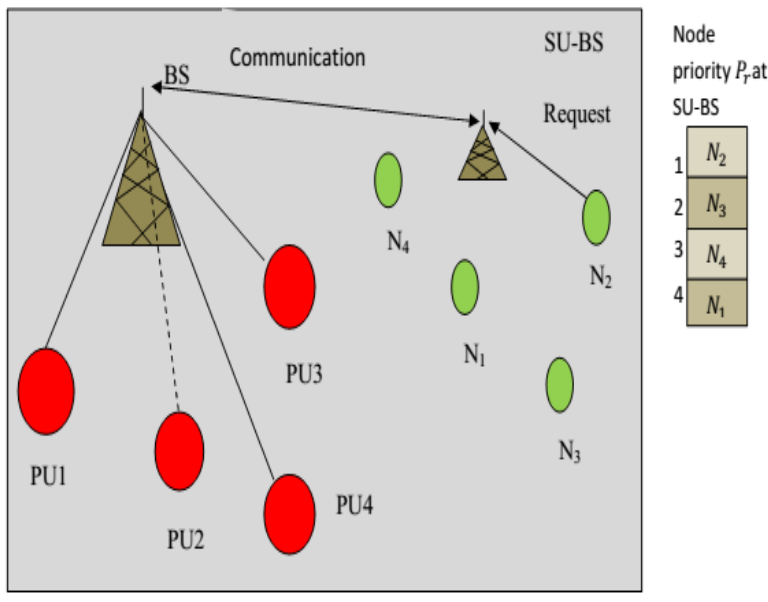

Figure.2 SU accessing the idle channel of PU through SU-BS

\section{Power}

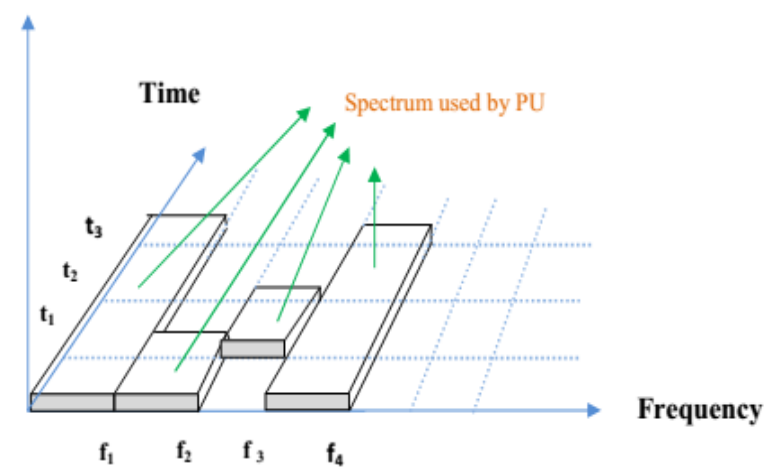

Figure.3 Illustration of spectrum Handoff

For each incoming stream, the priority of packets is determined depending on the service type and queuing delay [8]. Then an objective function is estimated for each stream by multiplying the priority with channel gain. Then the streams can be sorted in the descending order of this objective values, then allocated to the respective type of SU. The stream with highest objective function is allocated to the MRDG, followed by MRG and MDG. The stream with least objective function is assigned to $\mathrm{BE}$. Hence, as shown in fig. 2, $P_{r}$ value is assigned to each SU.

Existing Algorithm:
Step1: Define n sub-channels SUs $k_{1}$ and $k_{2}$
Step2: Sense the presence of PU
if yes
$\quad$ Hand-off
else
Step3: Free channel sensing and allocation to
SU
Step4: Sense the presence of PU
if yes
Hand-off
$\quad$ else
Step5: Power allocation to channel given to
SU
Step6: Sense the presence of PU
if yes
$\quad$ Hand-off
else
Step7: Data transmission takes place
Step 8: Sense the presence of PU
if yes
Hand-off
else
Step 9: Channel allocated to other SU
Step 10: Goto Step 2




\section{Simulation Result}

We use $n s-2$ simulation package to simulate the proposed algorithm using the cognitive radio. At the MAC layer, we use IEEE 802.11. The network field considered for the simulation is $1000 \mathrm{~m} \mathrm{X} 1000 \mathrm{~m}$ over a flat region for 70 seconds of simulation time. All nodes have the same transmission range of 250 meters. The simulated traffic is Constant Bit Rate (CBR) with a packet size of $512 \mathrm{~B}$. The simulation settings and parameters are summarized in Table1.

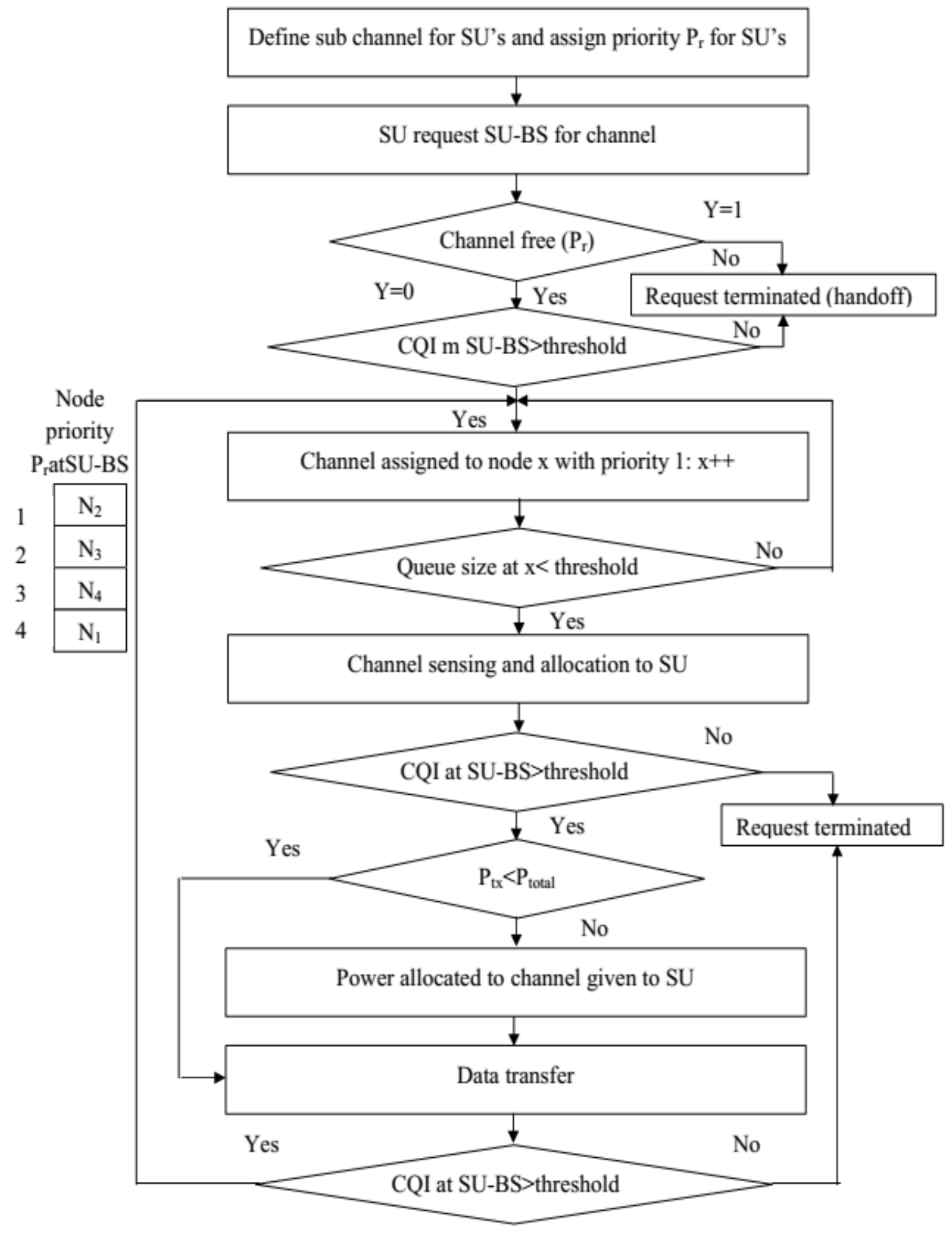

Figure.4 Proposed algorithm 


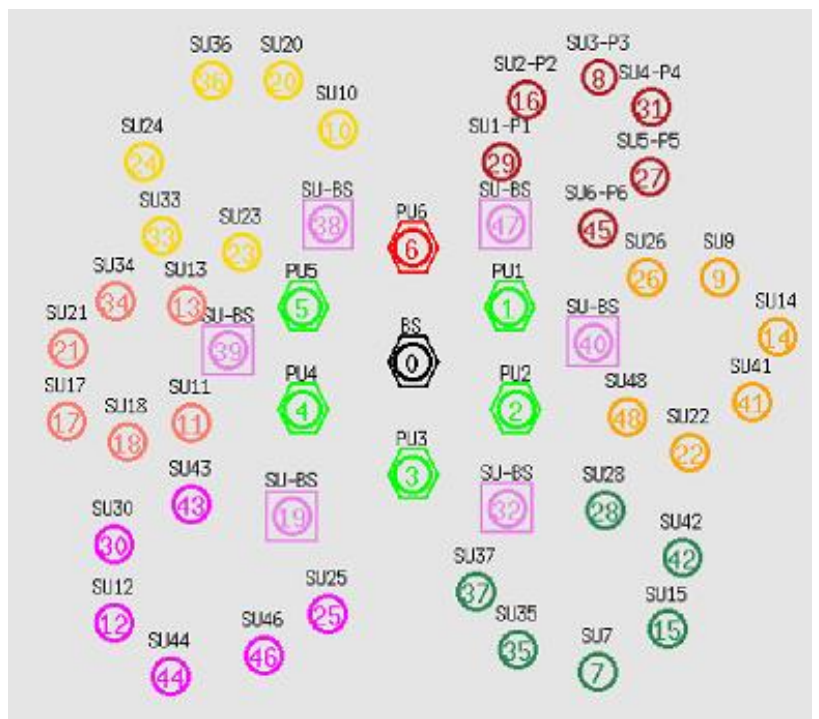

Figure.5 Screen shot of the out.nam for the proposed approach

Table 1. Simulation Environment

\begin{tabular}{|l|l|}
\hline No. of Nodes & $20,40,60,80$ and 100 \\
\hline Area Size & $1000 \mathrm{~m}$ X $1000 \mathrm{~m}$ \\
\hline Mac & IEEE802.11 \\
\hline Transmission Range & $250 \mathrm{~m}$ \\
\hline Simulation Time & $70 \mathrm{sec}$ \\
\hline Traffic Source & CBR \\
\hline Packet Size & $1000 \mathrm{~B}$ \\
\hline Sources & 4 \\
\hline Attackers & 2 \\
\hline Nodes Speed & $5,10,15,20$ and $25 \mathrm{~m} / \mathrm{s}$ \\
\hline
\end{tabular}

As shown in fig.1, six cognitive radio networks are considered with 6 SUs in each CRN. PU-6 is idle as shown in fig.5 and has channel-6 as idle.

The Proposed DRAPBS-SRN Algorithm is compared with DRA-CRN algorithm. As DRAPBSSRN employs dynamic resource allocation; it outperforms the traditional approaches in cognitive radio.

\section{Scenario 1: Quality and robustness of Channel allocation in DRAPBS}

Fig.6 shows the delay DRAPBS-SRN in comparison to DRA-CRN. With varying number of idle channels present at the PU-BS, the proposed approach is found to experience less delay since each SU is prioritized in accessing the idle channel without any interference occurring at the channel.

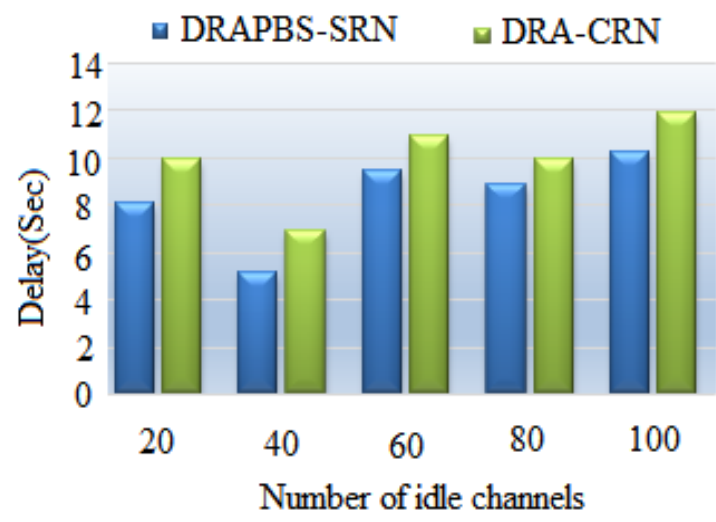

Figure.6 Channels Vs Delay

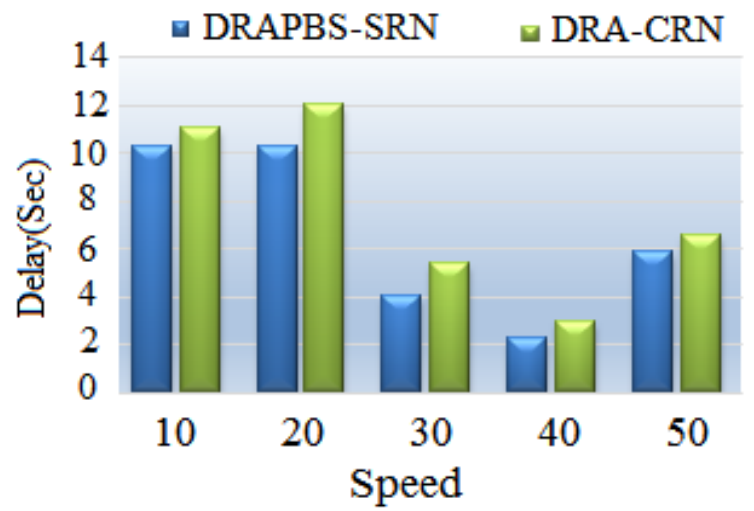

Figure 7. Speed Vs Delay

Since each SU senses the channel before accessing the channel and also periodically checks for the existence of the PU, the interference introduced due to the sudden appearance of PU is reduced. Prioritization of SU's at the SU-BS leads to idle channel allocation robustly. DSAPBS-SRN experiences $17 \%$ less delay compared to DRA-CRN approach. From fig. 7 it is also found that the delay experienced, due to varying range of mobility of nodes, is $16 \%$ less than DRA-CRN approach.

From fig. 8, the interference experienced by any idle channel for communication (along with tradeoff) is $9 \%$ less than DRA-SRN approach. From fig. 9, it can be observed that at varying scenario of mobility of nodes DSAPBS-SRN is found to be more stable compared to DRA-CRN. Interference in the proposed DRA-SRN approach is reduced due to the Channel Quality Indicator can be measured at SU-BS. Here channel is estimated and assigned to the SU only if the $C Q I>$ threshold. At mobility of 10 $\mathrm{m} / \mathrm{s}, 20 \mathrm{~m} / \mathrm{s}$ and $30 \mathrm{~m} / \mathrm{s}$ the interference experienced by the network due to the utilization of DSAPBSSRN is negligible compared to DRA-CRN by $58 \%$. 


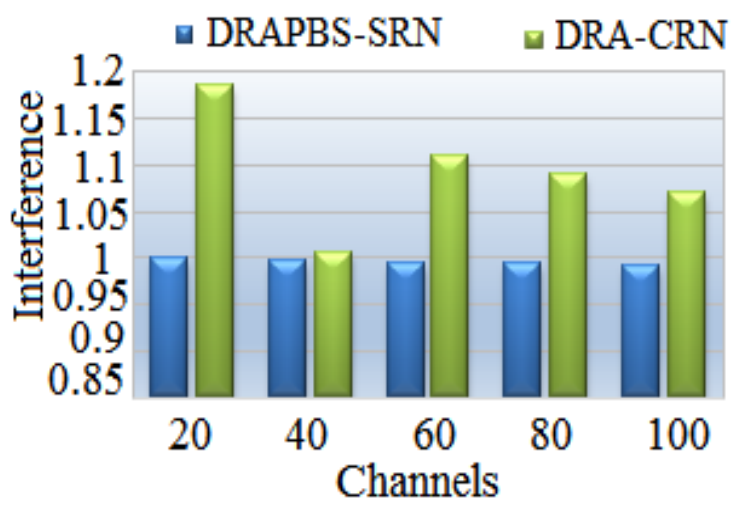

Figure.8 Channels Vs Interference

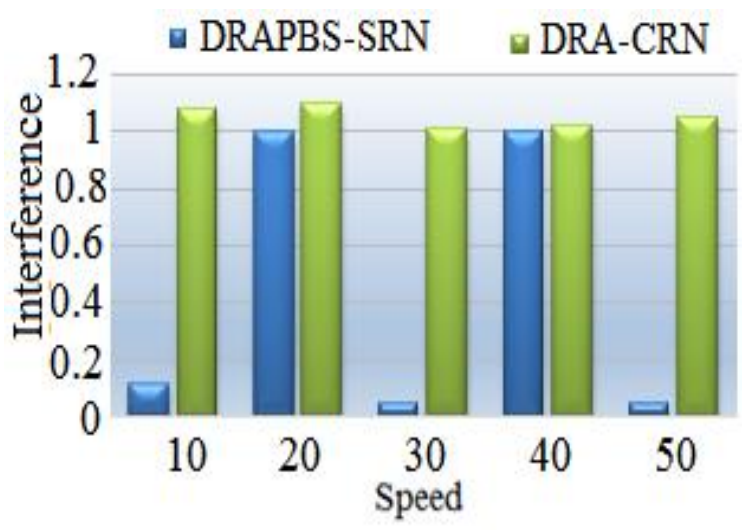

Figure.9 Speed Vs Interference

The inefficiency in resource allocation by the BS (for a given frequency band used by the PU) leads to interference at the channel leading to collision of data sent using the defined channel and hence the network experiences more drop of data packets in comparison to an efficient network with robust channel allocation and high delivery ratio. With total power constraints (as calculated in eq. (1)), minimum rate guarantee ( as calculated in eq. (2)) and fairness constraints (as calculated in eq. (3)), our proposed approach employs an efficient channel allocation procedure (as discussed in section 3), experimentally it is observed that DRAPBS-SRN is efficient in comparison to DRA-CRN.

\section{Scenario 2: Network Performance Evaluation}

In Fig. 10 and 11, drop refers the average number of packets dropped during the transmission. Each channel in the CRN experiences drop at each channel with respect to the number of idle channels and mobility of nodes present in the network.

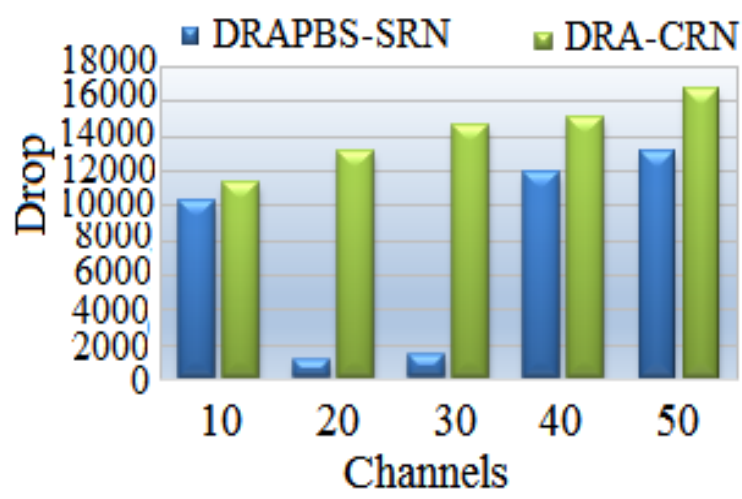

Figure.10 Channel Vs Drop

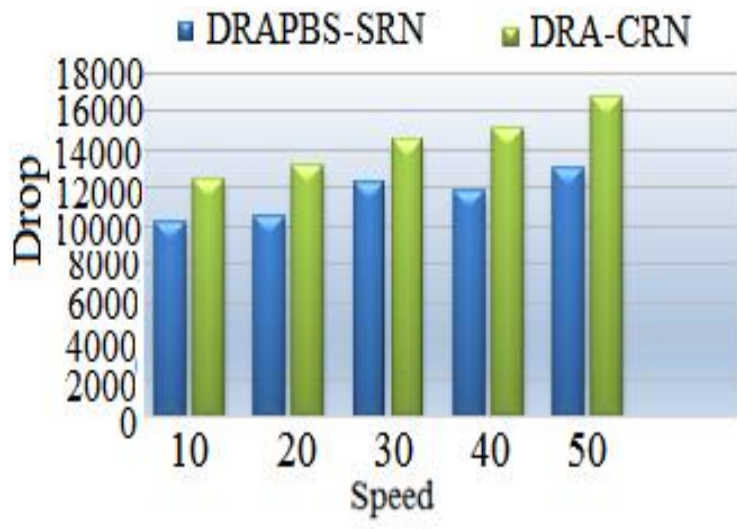

Figure.11 Speed Vs Mobility

As the number of idle channels in the network increases from 20 to 30 channels, the packet drop due to the employment of DRAPBS-SRN is found to be negligible as compared to DRA-CRN by $47 \%$. Due to prioritization of SU at the SU-BS, each idle channel in the network experiences less delay and more throughput. From fig. 12, it can be observed that DRAPBS-SRN is found to experience $19 \%$ of less drop in data packets in comparison to DRACRN.

Network throughput is the rate of successful message delivery over a communication channel. It is usually measured in bits per second (or data packets per second or data packets per time slot). Hence for an efficient and reliable network, the throughput must be maximum and it should experience less drop in the packet. As already observed from fig. 10 and fig. 11, drop of data packets is negligible in our proposed approach with soft spectrum handoff. Similarly, from fig 12, throughput in DRAPBS-SRN is $41 \%$ more efficient with varying the number of idle channels from 20 channels to 100 channels in the given network. 


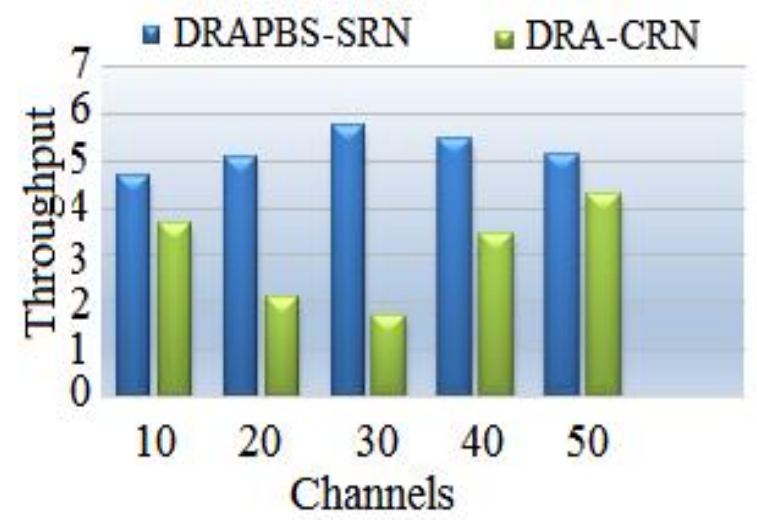

Figure.12 Channel Vs Throughput

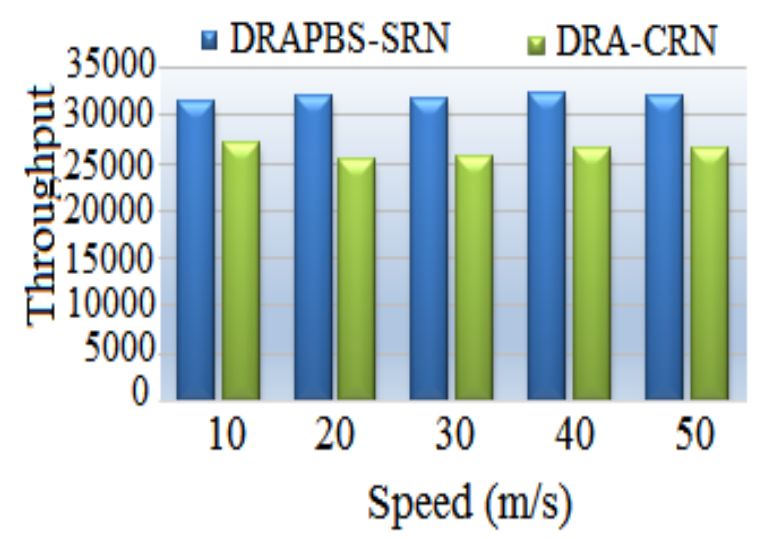

Figure.13 Speed Vs Throughput

As shown in Fig.13, with variations in the mobility of nodes from $10 \mathrm{~m} / \mathrm{s}$ to $50 \mathrm{~m} / \mathrm{s}$ the throughput of DRAPBS-CRN is found to be $3 \%$ efficient in comparison to DRA-CRN, with an efficient calculation of CQI.

\section{Conclusion}

However, when cognitive radio theories are becoming mature gradually and start to turn an interesting topic among researchers, lots of problems confronted in practical application are still challenging the realization of the Cognitive radio network. Our focus has been laid on optimum resource allocations and scheduling for primary user (PU) and secondary user (SU) to maximize the performance of the whole network by reducing the interference at $\mathrm{PU}$ and also maximizing the throughput and minimizing the risk of overlapping the coverage of CRNs. As a future work we would like to interface the proposed approach with artificial intelligent and human like decision making qualities, to enhance the performance during handoff and channel accessing. Prioritization of SUs can be made efficient by using a best optimization approach with higher convergence.

\section{Reference}

[1] P. Cheng, A. Guo, Y. Xu, X. Wang, and X. Gao, “A game approach for dynamic resource allocation in cognitive radio networks", Wireless Communications and Signal Processing (WCSP), International Conference on. IEEE, pp. 1-6, 2010.

[2] Y. Tachwali, B. F. Lo, I. F. Akyildiz and R. Agust, "Multiuser Resource Allocation Optimization Using Bandwidth-Power Product in Cognitive Radio Networks", IEEE journal on selected areas in communications, Vol. 31, No. 3, pp. 451-463, 2013.

[3] R. Xie, F. R. Yu, and H. Ji, "Spectrum Sharing and Resource Allocation for Energy-Efficient Heterogeneous Cognitive Radio Networks with Femtocells", IEEE ICC 2012 - Cognitive Radio and Networks Symposium, pp. 1661-1665, 2012.

[4] Y. Teng, Y. Liu and Y. Zhang, "An Energy Efficient Resource Allocation in Cognitive Radio Networks with Pairwise NBS Optimization for MultiSecondary Users", IEEE Wireless communication, pp. 744-749, 2013.

[5] M. G. Adian and H. Aghaeinia, "Optimal resource allocation for opportunistic spectrum access in multiple-input multiple-output orthogonal frequency division multiplexing based cooperative cognitive radio networks", IET Signal Process, Vol. 7, No. 7, pp. 549-557, 2013.

[6] J. L. Xu, M. Chen and N. Wang, "Optimal Cooperative Sensing and Resource Allocation in Cognitive Radio Networks", Wireless Communications \& Signal Processing (WCSP), International Conference on. IEEE, pp. 1-6, 2013.

[7] M. Alfonso, Jeison, and L. Betancur Agudelo. "Centralized Spectrum Broker and Spectrum Sensing with Compressive Sensing techniques for resource allocation in Cognitive Radio Networks." Communications (LATINCOM), 2013 IEEE LatinAmerica Conference on. IEEE, pp. 1-6, 2013.

[8] C. Gao, S. Chu, and X. Wang, "Distributed Scheduling in MIMO Empowered Cognitive Radio Ad Hoc Networks", IEEE transactions on mobile computing, Vol. 13, No. 7, pp. 1456-1468, 2014.

[9] D. Gozupek and F. Alagoz, "A Fair Scheduling Model for Centralized Cognitive Radio Networks", arXiv preprint arXiv: 1309.2233, 2013.

[10]Y. Li and A. Nosratinia, "Hybrid Opportunistic Scheduling in Cognitive Radio Networks", IEEE transactions on wireless communications, Vol. 11, No. 1, 328-337, 2012.

[11]H. Li, X. Cheng, Keqiu, X. Xing and T. Jing, "Utility-Based Cooperative Spectrum Sensing Scheduling in Cognitive Radio Networks", INFOCOM, 2013 Proceedings IEEE. IEEE, pp. 165169, 2013.

[12]R. Xie, F. R. Yu and H. Ji, "Dynamic Resource Allocation forheterogeneous Services in Cognitive Radio Networks with Imperfect Channel Sensing”, IEEE transactions on vehicular technology, Vol. 61, No. 2, pp. 770-780, 2012. 
[13]P. M. R. Santos, M. A. Kalil, O. Artemenko, A. Lavrenko, and A. M. Thiel, "Self-Organized Common Control Channel Design for Cognitive Radio Ad Hoc Networks", IEEE 24th International Symposium on Personal, Indoor and Mobile Radio Communications: Mobile and Wireless Networks, pp. 2419-2423, 2013.

[14]S. Byun, "TCP over scarce transmission opportunity in cognitive radio networks", Computer Networks, Vol. 103, pp. 101-114, 2016.

[15]K. Berbra, M. Barkat, F. Gini, M. Greco and P. Stinco, "A fast spectrum sensing for CP-OFDM cognitive radio based on adaptive thresholding", Signal Processing, vol. 128, pp. 252-261, 2016.

[16]U. Premarathne, I. Khalil and M. Atiquzzaman, "Trust based reliable transmissions strategies for smart home energy consumption management in cognitive radio based smart grid", Ad Hoc Networks, vol. 41, pp. 15-29, 2016.

[17]V. Kumar, S. Minz and V. Kumar, "Performance analysis of cognitive radio networks under spectrum sharing using queuing approach", Computers \& Electrical Engineering, 2016.

[18]C. Charan and R. Paney, "Eigenvalue based double threshold spectrum sensing under noise uncertainty for cognitive radio", Optik - International Journal for Light and Electron Optics, Vol. 127, No. 15, pp. 5968-5975, 2016.

[19]M. Khasawneh, S. Alrabaee, A. Agarwal, N. Goel and M. Zaman, "Power trading in cognitive radio networks", Journal of Network and Computer Applications, Vol. 65, pp. 155-166, 2016.

[20]O. Ergul, A. Bicen and O. Akan, "Opportunistic reliability for cognitive radio sensor actor networks in smart grid", Ad Hoc Networks, Vol. 41, pp. 5-14, 2016.

[21]M. Moon and V. Gulhane, "Appropriate Channel Selection for Data Transmission in Cognitive Radio Networks", Procedia Computer Science, Vol. 78, pp. 838-844, 2016.

[22]V. Esmaeelzadeh, E. Hosseini, R. Berangi and O. Akan, "Modeling of rate-based congestion control schemes in cognitive radio sensor networks", Ad Hoc Networks, Vol. 36, pp. 177-188, 2016.

[23]A. Sharifi, M. Sharifi and M. Niya, "Secure cooperative spectrum sensing under primary user emulation attack in cognitive radio networks: Attackaware threshold selection approach", $A E U$ International Journal of Electronics and Communications, Vol. 70, No. 1, pp. 95-104, 2016.

[24]G. Zhang, X. Li, M. Cui, G. Li and Y. Tan, "Transceiver design for cognitive multi-user MIMO multi-relay networks using imperfect CSI", $A E U$ International Journal of Electronics and Communications, Vol. 70, No. 5, pp. 544-557, 2016.

[25]S. Maity, S. Chatterjee and T. Acharya, "On optimal fuzzy c-means clustering for energy efficient cooperative spectrum sensing in cognitive radio networks", Digital Signal Processing, Vol. 49, pp. 104-115, 2016. 